\title{
A NOVEL DESIGN CONCEPT FOR A THERMALLY STABLE LINEAR SCALE USING TWO DIFFERENT MATERIALS
}

\author{
T. H. Lee ${ }^{1 *}$, H. Gim¹ , S. Oh¹, T. Gotthardt' F. Tzanetos ${ }^{1}$, A. Schmetz ${ }^{1}$, D. Zontar ${ }^{1}$, C. Brecher ${ }^{1,2}$ \\ ${ }^{1}$ Fraunhofer Institute for Production Technology IPT, Steinbachstrasse 17, 52074 Aachen, Germany \\ ${ }^{2}$ Laboratory for Machine Tools and Production Engineering (WZL), Campus-Boulevard 30, 52074 Aachen, Germany \\ ${ }^{\star}$ Corresponding author; e-mail: tae.hun.lee@ipt.fraunhofer.de
}

\begin{abstract}
Linear scale has significant impacts on the machine tool accuracy, since the positioning of the linear axes are controlled by its measurement. This paper presents a novel concept of linear scale design which can provide high thermal stability with low cost. This concept applies two different materials: a steel linear scale attached mechanically on a carbon fiber reinforced plastic (CFRP) tube. Attaching this two materials, the thermal behavior of the steel scale can be mechanically compensated by the CFRP tube when the temperature changes. The potential of the design concept is analyzed based on the experiment results.
\end{abstract}

\section{Keywords:}

Linear scale; Hybrid material; Thermal behavior; Carbon fiber reinforced plastic

\section{INTRODUCTION}

Increased demand for ultra-precision and efficiency in the metal-cutting industry in the past 20 years have led to extensive research in the field of thermo-elastic behavior of machine tools. Up to $75 \%$ of geometrical workpiece errors can be traced back to thermal issues in machine tools [Mayrs 2012]. In industry, linear scale encoders demand high thermal stability so that error propagation to the workpiece could be minimized. Hence, glass or glass ceramics are often used due to their low coefficient of thermal expansion (CTE).

The following describes a novel combination of lower cost steel and CFRP for the linear scale design, as opposed to the current industry standard like Zerodur, which are costinhibitive. Steel has a positive CTE and CFRP, depending on the fiber directions and number of plies, a near-zero or negative CTE. By mechanically attaching these materials together, the experiment sought to investigate its therma stability for the purpose of precise scale measurements.

Thus in the following section, an overview of the technical developments of the thermally stable linear scales are presented (section 2). In section 3, our design concept for a scale with a theoretical zero CTE is proposed. Finally, the experimental setup for the thermal analysis of the developed scale (section 4) is presented, followed by the results and conclusion (section 5 ).

\section{TECHNICAL BACKGROUND}

Wingate first introduced the concept of encrypting linear movement by electrical output signals in linear motion encoders in 1970 [Wingate 1970]. Although DiCiaccio sought to improve its construction by relying on Guttmann's approach based on torque spring motors, it was conclusively more inaccurate, more complex, more expensive and problematic when used on different materials [DiCiaccio 1974]. In attempts to lessen the thermal effects of the materials used in their construction, Ichikawa developed a linear scale with a hollow elongate case [Ichikawa 1985].

At the same time, demand for low thermal expansion materials in electronic devices, heat-engine components, space telescopy and other industry had sparked new interest in various combinations of materials. Among them, $\mathrm{SiO}_{2}-\mathrm{TiO}_{2}$ glasses (pyrex, fused-silica), Zerodur, Invar (Fe-Ni alloy) and ferroic ceramics had shown either zero or negative CTE [Roy 1989]. Both Zerodur and Invar showed near-zero expansion ( $\alpha=0.05,0.07 \mu \mathrm{m} / \mathrm{m} / \mathrm{K})$ at room temperature, which is the typical environmental temperature for machine tool components.

In 1998, Spanner combined the use of these low CTE materials in the linear encoder [Spanner 1998]. Soon thereafter, Tondorf improved upon Spanner by decoupling the scale from its mounting fixture so that the deformation of the mount are not transmitted to the scale [Tondorf 2003]. Indeed, the thermal behavior of linear scales proved to be crucial to the accuracy of the finished workpiece. Alejandre 
and Artes have found in experiments that the thermal effects from linear scales can cause workpiece errors ten or one hundred times greater than other sources of error [Alejandre 2003]. Thus, it is paramount that deviations due to thermal expansion be limited as much as possible.

However, Zerodur and Invar are several orders of magnitude more expensive than other low-CTE materials such as CFRP. Hence, in machines with multiple axis, linear scales using these materials quickly become costinhibitive. CFRP, on the other hand, are also known to be thermally very stable at room temperature. In addition, they can be manufactured to have different thermal properties depending on the fiber direction, number of plies, fiber volume ratio, type of epoxy, type of fiber and curing process. For example, Joven demonstrated for 977-2 unidirectional fiber, a negative secant CTE of $-0.81 \mu \mathrm{m} / \mathrm{m} / \mathrm{K}$ was observed from 0 to $150{ }^{\circ} \mathrm{C}$ along the longitudinal direction [Joven 2012]. When arranged in different directions (i.e. weave), the CTE can also be controlled to be less negative or slightly positive. Thus, in combination with existing linear scales made of cheaper material (i.e. steel), CFRP presents itself as a cheaper thermally stable material than current industry standard materials that is more flexible in its use cases in linear scales.

\section{SCALE DESIGN}

The new scale design consists of an industrially suitable and inexpensive steel scale that is pretensioned longitudinally by a CFRP beam with a suitable stiffness and CTE (Fig. 1). Since scales are usually fixed on one side, they are not distorted under thermal load.

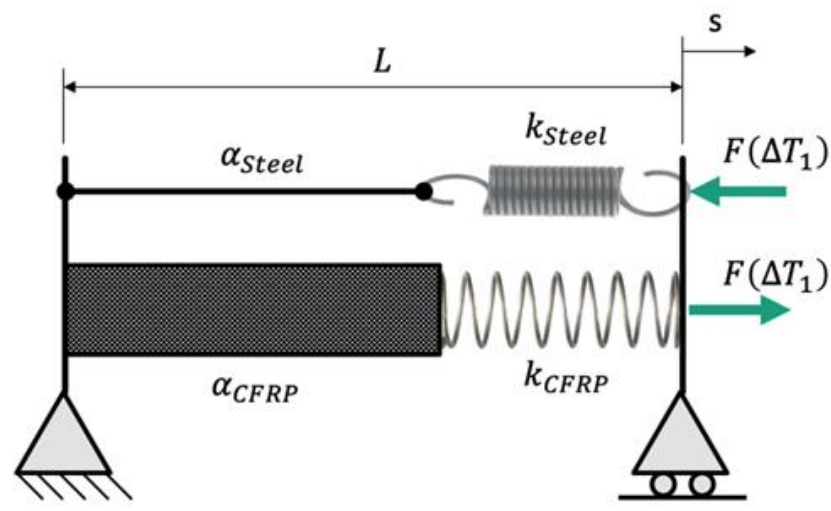

Fig. 1: Schematic design of the CFRP-Steel scale with the description of its expansion caused by different CTE of steel and CFRP

When heated by $\Delta T_{1}$, both materials generate a therma expansion component $\alpha \cdot L \cdot \Delta T_{1}$ as well as a resultant mechanical force component $k \cdot \Delta \mathrm{l}$ due to the differing thermal expansion of two materials, where $L$ is the length and $\Delta \mathrm{l}$ is the deformation of each component (Fig. 2, Eq. $1 \mathrm{a}, \mathrm{b})$. Since the steel scale has a larger CTE than CFRP, there is a mechanical force caused by thermal deformation on CFRP. The equilibrium of forces for each material results in the deformation based on the assembly length, temperature change, the stiffness constant and CTE for each material (Eq. 2, 3).

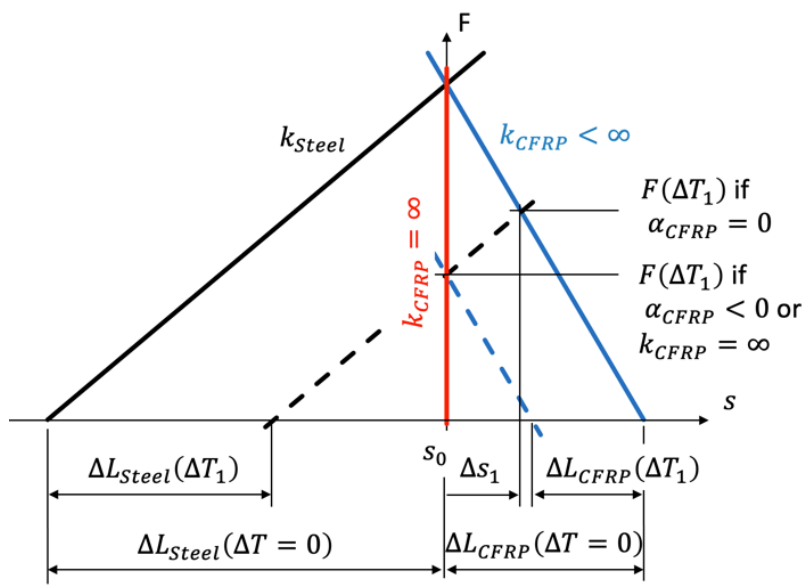

Fig. 2: Theoretical approach for the zero expansion scale using steel with a positive CTE and CFRP with a negative CTE

In the ideal case, an infinitely stiff CFRP do not allow deformation. However, on closer examination of Eq. 1, it is evident that a negligible CTE of CFRP can lead to a deformation $\Delta s_{1} \neq 0$. On the other hand, a correspondingly negative CTE of CFRP would theoretically enable the desired zero deformation, leading to a simple relationship only based on only the CTE and stiffness of the material (Eq. 4).

$\Delta l_{C F R P, T}=\Delta T \cdot \alpha_{C F R P} \cdot L$

$\Delta l_{\text {Steel,T }}=\Delta T \cdot \alpha_{\text {Steel }} \cdot L$

$F=k_{\text {Steel }}\left(\Delta s-\Delta l_{\text {Steel,T }}\right)=k_{C F R P, T}\left(\Delta l_{C F R P}-\Delta s\right)$

$\Delta s_{1}=\frac{k_{\text {Steel }} \alpha_{\text {Steel }}+k_{C F R P} \alpha_{C F R P}}{k_{\text {Steel }}+k_{C F R P}} \cdot L \cdot \Delta T_{1}$

$\frac{k_{\text {Steel }}}{k_{\text {CFRP }}}=\frac{\alpha_{\text {CFRP }}}{\alpha_{\text {Steel }}}$

To analyze the potential of this designed concept, a prototype scale was developed with a steel scale and a CFRP tube. Due to the financial constraints and the prototype first needing to be a proof-of-concept, a semifinished square CFRP tube was purchased with dimensions 300 (length) $\times 30 \times 30 \mathrm{~mm}$ with a thickness of $2 \mathrm{~mm}$. The profile was square with rounded corners with an estimated $1 \mathrm{~mm}$ corner radius. The composite contained three layers of carbon fabric with fibers in different directions: 1$)+/-45^{\circ}$ for torsional stability 2) $0^{\circ}$ unidirectional for longitudinal strength and 3) $0 / 90^{\circ}$ plain weave for resistance against bending. The cost of the CFRP tube was under $\$ 100$. Since semi-finished CFRP products are usually not intended for use in thermal-sensitive tasks, many providers do not measure the CTE of their products. However, based on the given data from the manufacturer and literature, our CFRP tube is estimated to have CTE between -0.76 and 4.02 $\mu \mathrm{m} / \mathrm{m} / \mathrm{K}$ in the longitudinal direction [Joven 2012].

The procured steel scale was a general commercial scale which has the measurement length of $300 \mathrm{~mm}$ and CTE $\alpha$ of $10 \mu \mathrm{m} / \mathrm{m} / \mathrm{K}$. The profile was rectangular with dimensions $16 \mathrm{~mm} \times 5.9 \mathrm{~mm}$. Using equations (3) and the properties of both materials, the CFRP-steel scale was estimated to have a CTE between -0.22 and $4.33 \mu \mathrm{m} / \mathrm{m} / \mathrm{K}$.

For comparison, a high quality Zerodur scale was also installed, which has near-zero CTE with the measurement length of app. $250 \mathrm{~mm}$. 


\section{EXPERIMENTAL SETUP}

The entire assembly including the CFRP-steel combination along with a reference Zerodur scale is pictured in the rendering below (Fig. 3). The metal scale was attached to the mounts via bolts on both sides. The CFRP profile, on the other hand, was glued to the mounts on either side. In other words, there were no direct mechanical interaction between CFRP and the steel scale, minimizing any deformation due to contact between each element. To ensure straightness, guides were placed beneath the CFRP profile to minimize movement in the latitudinal direction. Heating was supplied via a heating element which increased temperature along a heat plate located in the middle of the assembly. The thermal behavior of the prototype was compared with that of the high quality Zerodur scale under simple linear temperature profiles up to a predefined temperature.

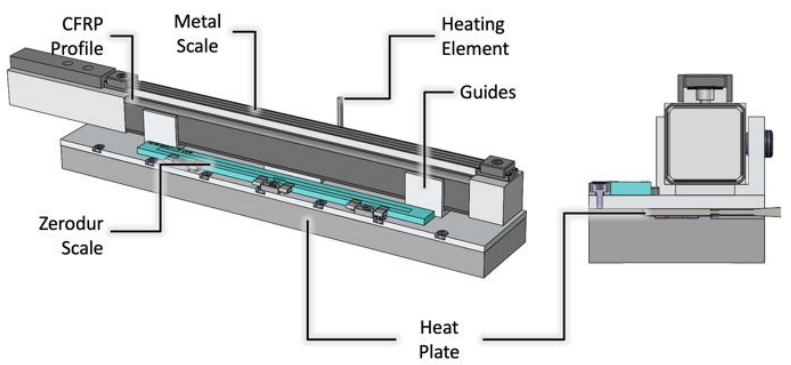

Fig. 3: Design of the test bench for the analysis of CFRPsteel scale in comparison to the Zerodur scale

The setup in close view is shown in Fig. 4. Both scales were integrated on a test bench made of granite and a linear axis. The scale was fixed along the longitudinal axis via the guides. Note that the guides were installed to minimize movement in any other direction except the linear axis. Deformation in axes other than longitudinal were not measured because their influence on the length measurement of the scale could be neglected.

Along the axis, an interferometer was installed to measure absolute distance for comparison with what the scales measure. The reflector for the laser interferometer was mounted on the moving part of the axis. The setup viewed from the top reveals the heating plate and the temperature sensors, positioned in the middle and both ends of the assembly. A single heat plate introduced temperature increases to both scales which were installed in parallel. The room where the test bench was located was temperature controlled at $20 \pm 0,5^{\circ} \mathrm{C}$ using air conditioning systems.

For analysis, two experiments were defined using different thermal loads with two different heating profiles: 1) 80 minutes of heating and 2) 120 minutes of heating. After constant thermal input, the test bench was cooled at the room temperature of $20{ }^{\circ} \mathrm{C}$ for over 10 hours. Before each experiment, the test bench was cooled down over 12 hours to assure the relatively homogenous temperature field of the test bench.

During both experiments, the assembly was moved along its longitudinal axis repeatedly from $0 \mathrm{~mm}$ to $200 \mathrm{~mm}$ back and forth. The assembly was stopped every $20 \mathrm{~mm}$ for absolute distance measurement by the laser interferometer as well as both of the scales. The deviation between the measured axis movement with the laser interferometer and scales was recorded as the thermal expansion of each scale.
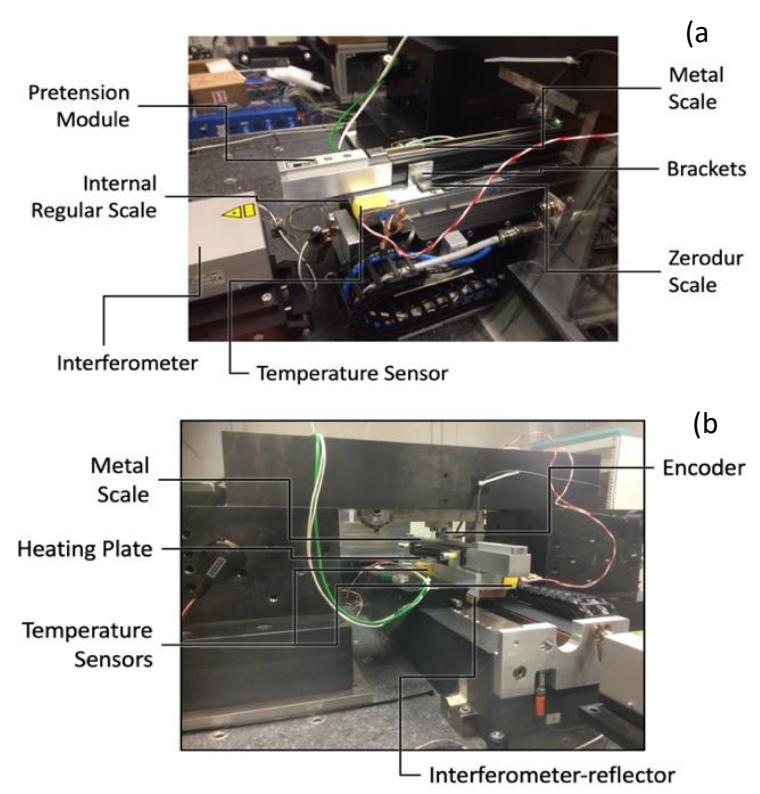

Fig. 4: Setup of the test bench from an upper view (a) and side view (b)

\section{EXPERIMENT RESULT}

Fig. 5 shows the measured temperature over two experimental setups. Since the environment room of the test bench was kept constant at $20^{\circ} \mathrm{C}$, the temperature of the sensors remained consistent with the environment at the beginning of the experiment. The first experiment applied heat to the assembly for 120 minutes. The temperature was raised to $34^{\circ} \mathrm{C}$ according to the sensor, resulting in a total temperature change of $14^{\circ} \mathrm{C}$.

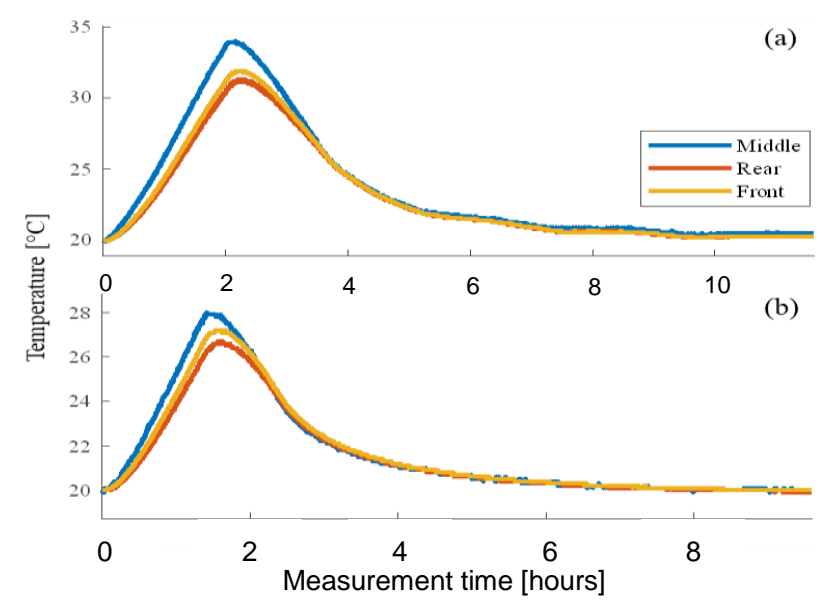

Fig. 5: Measured temperature changes of the test bench over experiments. (a): 120 minutes of heating, (b): 80 minutes of heating

The second experiment heated the plate for 80 minutes and a maximum temperature change of $8{ }^{\circ} \mathrm{C}$ was measured. The temperature was measured to lower back to approximately $20^{\circ} \mathrm{C}$ each after 8 hours and 6 hours of cooling, respectively.

The different profiles of the measured temperature are dependent on their position relative to one end of the test bench. Since the middle sensor was positioned very near 
the heat source, it measured the greatest temperature throughout both experiments. The rear sensor, which is placed near the rear side of the CFRP-steel scale, measured the lowest temperature. The measured difference of three temperature sensors did not exceed $3{ }^{\circ} \mathrm{C}$ at any point.

The two diagrams in Fig. 6 exhibit the measurements of thermal expansion at two different axis positions in the first experiment; (a) $100 \mathrm{~mm}$ and (b) $200 \mathrm{~mm}$. The measurements in each of the three temperature sensors were averaged to create the diagrams.
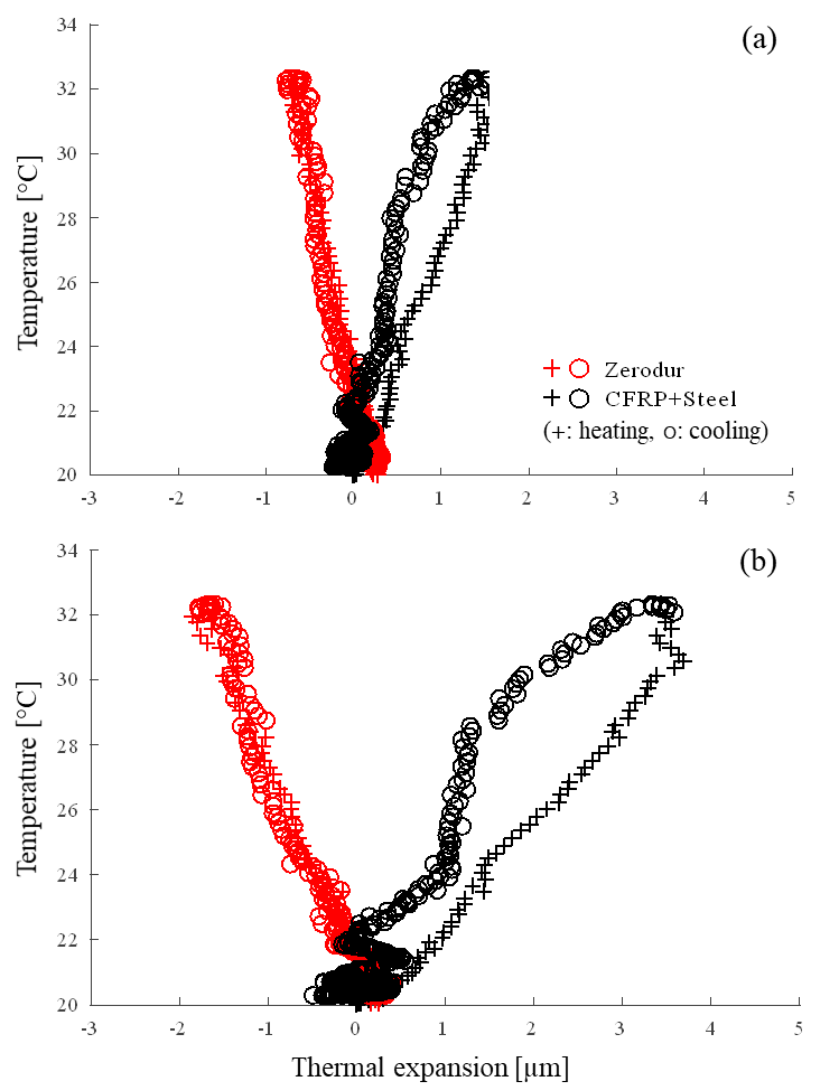

Fig. 6: Measured thermal expansion of the linear scales at $100 \mathrm{~mm}$ (a) and $200 \mathrm{~mm}$ (b) during 120 minutes heating

In diagram (a), the Zerodur scale expanded in the negative direction up to $-0.8 \mu \mathrm{m}$. The gradient for expansion during heating and cooling stages almost coincide, exhibiting restorative properties. The CFRP and steel scale showed positive thermal expansion up to $1.5 \mu \mathrm{m}$ over the temperature change of over $12^{\circ} \mathrm{C}$.

The hysteresis of the slopes from heating and cooling can be observed. This can be attributed due to the inhomogeneous temperature field on the CFRP structure and the steel scale. Since the CFRP-steel scale is relatively large and the heating was applied with a heat plate from only the bottom side, an inhomogeneous temperature development could have developed in the overall structure. In addition, the cooling was due to the exothermic process due to the thermal bath at $20{ }^{\circ} \mathrm{C}$, or room temperature. Therefore, cooling could have affected the structure differently depending on the accessibility and the heat transfer coefficient of different components. These effects are negligible for the Zerodur scale, since the scale is relatively small and made of one material.

At the measurement position of $200 \mathrm{~mm}$, the Zerodur scale and the CFRP-steel scale showed similar expansion trends as at the position of $100 \mathrm{~mm}$, with approximately doubled maximum expansions. The Zerodur scale showed negative expansions up to $-2 \mu \mathrm{m}$ and the CFRP-steel scale positive expansions up to $3.7 \mu \mathrm{m}$ during the course of the experiment. The CFRP-steel scale measurements showed larger hysteresis at $200 \mathrm{~mm}$ than at $100 \mathrm{~mm}$, since the influence of the inhomogeneous temperature field was exaggerated over the longer length of the structure.

The measurement results of the second experiment showed similar trends (Fig. 7) as in the first experiment. The small hysteresis of the Zerodur can be caused by averaging of the temperature values from three sensors, since the sensors have slightly different values due to temperature inhomogeneity. For the CFRP-steel scale, the expansion after the cooling differs slightly from it at the beginning of the experiment. This could be caused by not enough tempering time before the experiment began. However, similar thermal expansion trends were observed for both scales as at the first experiment.

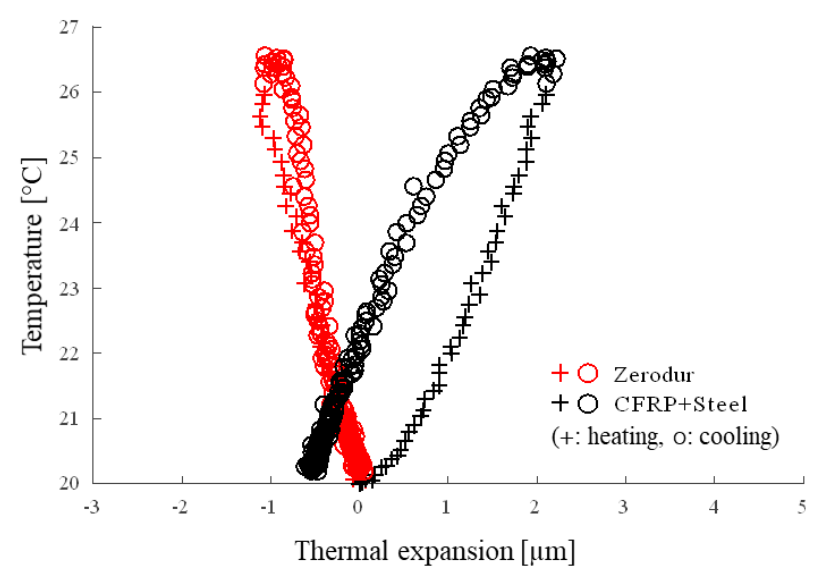

Fig. 7: Measured thermal expansion of the linear scales at $200 \mathrm{~mm}$ for the experiment of 80 minutes heating

Based on the measurement results of two experiments, the CTE of the scales were analyzed (Tab. 1). For this, the trend of the measured slopes were calculated at each measurement positions. The Zerodur scale showed the CTE between -0.6 to $-0.76 \mu \mathrm{m} / \mathrm{m} / \mathrm{K}$ and the CFRP-steel scale between 1.15 to $1.43 \mu \mathrm{m} / \mathrm{m} / \mathrm{K}$.

However as mentioned, the CFRP-steel structure was heated from the bottom side and was significantly larger than the Zerodur scale. Thus, the temperature over the structure might have differed and the inhomogeneous temperature field could have affected the thermal expansion. Nevertheless, the CFRP-steel shows potential for the further research, since the calculated CTE is very low for the applied material cost which is significantly lower than the Zerodur scale and even lower than the other alternative materials such as glass or invar.

Tab. 1: Measured thermal expansion coefficient of the scale

\begin{tabular}{|l|l|l|l|}
\hline \multirow{2}{*}{ Ex. } & \multirow{2}{*}{$\begin{array}{l}\text { Axis } \\
\text { position }\end{array}$} & \multicolumn{3}{|l|}{ Thermal expansion $[\mu \mathrm{m} / \mathrm{m} / \mathrm{K}]$} \\
\cline { 3 - 4 } & & Zerodur & CFRP-steel \\
\hline \multirow{2}{*}{1} & $100 \mathrm{~mm}$ & -0.60 & 1.24 \\
\cline { 2 - 4 } & $200 \mathrm{~mm}$ & -0.69 & 1.43 \\
\hline \multirow{2}{*}{2} & $100 \mathrm{~mm}$ & -0.61 & 1.15 \\
\cline { 2 - 4 } & $200 \mathrm{~mm}$ & -0.76 & 1.15 \\
\hline
\end{tabular}




\section{CONCLUSION AND OUTLOOK}

In this paper, a concept of a thermally stable scale is proposed using two different materials-CFRP and steel. Depending on the fiber directions, the CFRP exhibit negative thermal expansion coefficients. Thus, using an appropriate CFRP structure, the positive thermal expansion of the steel scale can be mechanically compensated. A calculation method for the theoretical zero expansion scale is introduced using the physical properties of the materials.

Based on the theory, a scale was made by a commercial CFRP tube and a steel scale with $200 \mathrm{~mm}$ measurement length. The steel scale was attached mechanically on the CFRP tube at its both ends. A test bench was made for the analysis of the performance of this hybrid scale. For comparison, a high quality Zerodur scale was installed in parallel to the CFR-steel scale. This test bench was made of an axis, a heat plate and a laser interferometer. The heat plate was placed under both scales. Using the laser interferometer as the absolute scale, the thermal expansion of both scales were measured and analyzed.

Two experiments were carried out using differing durations of heat application. The expansion of two scales were measured at two different axis positions. The Zerodur scale showed negative thermal expansion coefficients between - 0.6 and $-0.76 \mu \mathrm{m} / \mathrm{m} / \mathrm{K}$, while the CFRP-steel scale showed between 1.15 and $1.43 \mu \mathrm{m} / \mathrm{m} / \mathrm{K}$. However, the CFRP-steel scale showed also hysteresis in their thermal expansion between the heating and cooling stages. The reason is attributed to inhomogeneous temperatures, since the CFRP-steel scale is relatively large and the heating was applied from a single heat plate from the bottom side of the scale. Additionally, different cooling speeds due to differing material properties and structural accessibility could have caused temperature inhomogeneity as well. Finally, the specific thermal properties for the semi-finished CFRP used were not known, causing our estimates to be based on research on similar CFRP profiles. In spite of these possible errors, our measured CTEs for the CFRP-steel scale were well within our estimates (see section 3). Hence, it shows great potential in future research.

Therefore, future work could concentrate on the further analysis of the thermal behavior of the CFRP-steel scale. Different temperature variation profiles using environmenta temperature variation or more complex thermal states including extreme conditions could be applied. For example, the rapid temperature variation of the machine tool can be simulated with a step function. For the accurate analysis of the thermal behavior, the temperature should be measured at more positions on the scale structure. To validate its industrial application potential, a cost performance analysis is required. For this the CFRP-steel scale should be compared with additional scales such as glass or Invar scales. A more functional CFRP-steel scale could also be designed with a CFRP structure with a more appropriate CTE. Since the CTEs of the commercial product are often not given, this can be analyzed upon studies of the CFRP structure, e.g. with finite element analysis. In such a case, the feasibility and the cost of the realization must be considered for it to be applicable in industry. Indeed, the challenge lies also in the economical production of CFRP components with the desired CTE and stiffness, the realization of a thermally dynamic reliable connection between the two materials and an accurate pretensioning of not only the steel scale and the CFRP carrier but the entire assembly carrying these components together.

\section{REFERENCES}

[Alejandre 2004] Alejandre, I., and Artes, M. Machine tool errors caused by optical linear encoders. Proceedings of The Institution of Mechanical Engineers Part B-journal of Engineering Manufacture - PROC INST MECH ENG B-J ENG MA, 218. pp. 113-122. DOI: 10.1243/ 095440504772830255.

[Bayer-Helms 1985] Bayer-Helms, F., Darnedde, H., and Exner, G. Laengenstabilitaet bei Raumtemperatur von Proben der Glaskeramik "Zerodur". Metrologia, 1985, Vol. 21 , Iss. 49.

[Both 2013] Both, J. C. Tragfaehigkeit von CFK-MetallLaminaten unter mechanischer und thermischer Belastung. Munich: Technical University of Munich, Faculty of Mechanical Engineering, 2013.

[DiCiaccio 1974] DiCiaccio, J. J., et al. Linear Encoder. US Patent. 3791037, filed Feb. 17, 1972, issued Feb. 12, 1974.

[Ichikawa 1985] Ichikawa, S. Linear Scale Type Displacement Measuring Instrument. US Patent. 4492033 filed Apr. 4, 1983, issued Jan. 8, 1985.

[Joven 2012] Joven, R. Study of Thermal Expansion in Carbon Fiber Reinforced Polymer Composites. SAMPE International Symposium Proceedings, Charleston, South Carolina, United States of America, Jan. 2012.

[Mayr 2012] Mayr, J., et al. Thermal Issues in Machine Tools. CIRP Annals - Manufacturing Technology, 2012, Vol. 61, Iss. 2, pp. 771-791.

[Li 2016] Li, S. et al. Effect of Temperature Variation on Bond Characteristics between CFRP and Steel Plate. International Journal of Polymer Science, Vol. 2016, Art. 5674572

[Roy 1989] Roy, R., Agrawal, D. K., and McKinstry, H. A. Very Low Thermal Expansion Coefficient Materials. Annual Reviews of Material Science, Vol. 19, Iss., 19-81, pp. 5981.

[Spanner 1998] Spanner, E., and Pechak, P. Linear Encoder. US Patent. 5711084, filed Apr. 1, 1996, issued Jan. 27, 1998.tem

[Tondorf 2003] Tondorf, S. et al. Linear Measuring Device. US Patent. 6571486 B1, filed Nov. 24, 1999, issued June 3, 2003

[Wingate 1970] Wingate, S. A. 1970. Linear Motion Encoder. US Patent. 3533703, filed June 23, 1966, issued Oct. 13, 1970. 